\title{
Environmental responses mediated by histone deacetylation - biological and clinical implications
}

\section{Yichi (Tony) Zhang}

\author{
Carleton University
}

\section{Introduction}

The term epigenetics refers to processes that lead to heritable changes in the expression of genes without changes in the sequence of DNA1. These alterations in gene expression are a result of modifications made to chromatin and nonchromatin proteins (e.g. transcription factors). Hence, not only do epigenetic modifications play a role in many, if not all, biological processes, such as cell cycle control ${ }^{2}$ and disease ${ }^{3}$ (Figure 1), they also add a layer of complexity to gene expression above what is already known at the genomic level.

Epigenetic modification of chromatin proteins alters the ability of the transcriptional apparatus to bind and transcribe DNA, this altering gene expression. In the case of the chromatin protein histone ${ }^{3}$, acetylation has been shown to lead to transcriptional activation, whereas deacetylation has been shown to result in transcriptional repression. Therefore, numerous studies have been conducted on histone deacetylases (HDACs) in association with transcriptional silencing ${ }^{4,5,6}$. Upon further observation, HDACs have also been shown to modulate the activities of various transcription factors and non-histone proteins, thus allowing for even greater precision in the regulation of gene expression ${ }^{3}$ (Figure 1). The present review will discuss the affect of environmental conditions on histone deacetylation, its implications for disease, and the use of HDAC inhibitors as a potential therapeutic for a multitude of diseases.

\section{Histone Deacetylation and Environmental Response}

Any organism is subject to environmental and physiological

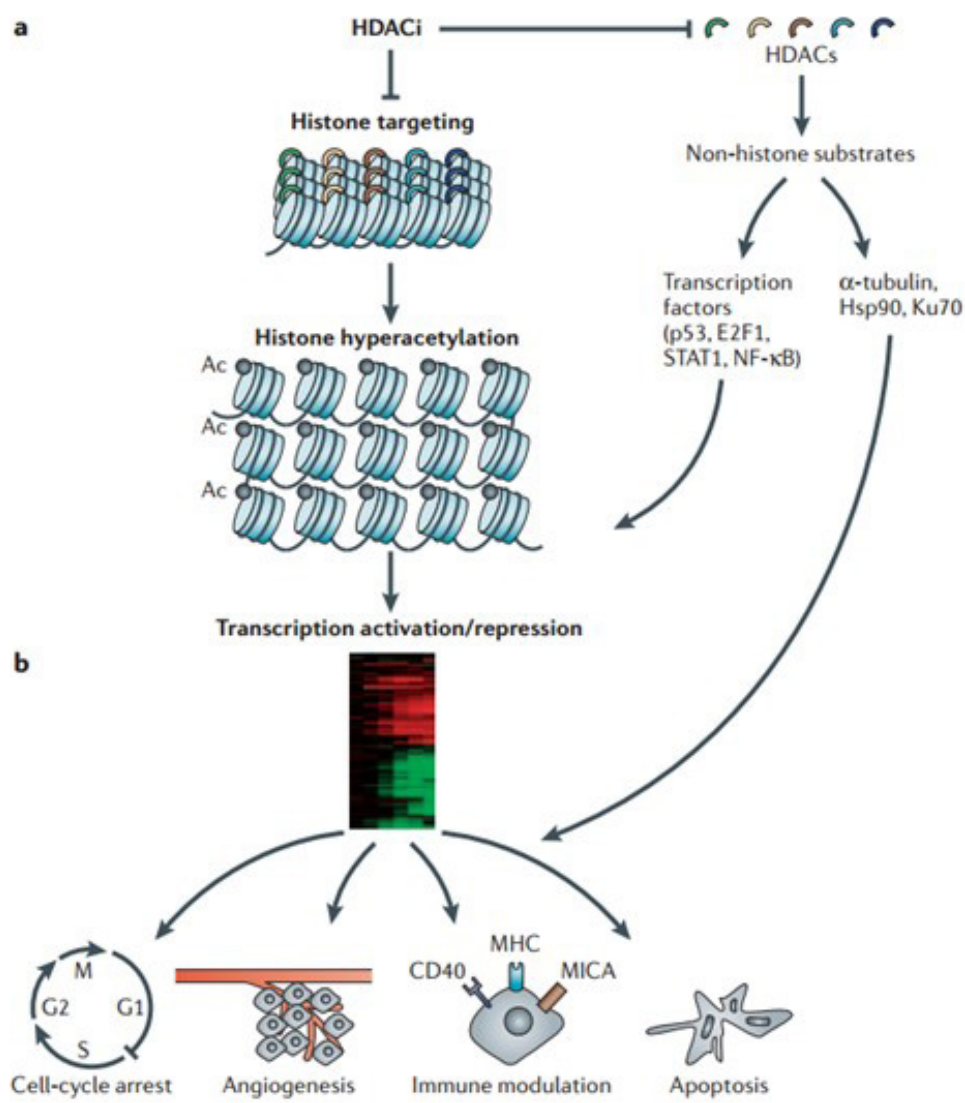

Figure 1. This diagram illustrates the mechanism by which HDACs regulate the acetylation state of histone and non-histone proteins to regulate gene expression. The effect of HDAC inhibitors on many biological processes is also shown. Figure Adapted from Bolden et al. ${ }^{3}$

stresses that puts them at risk. With regard to environmental factors (e.g. temperature, oxygen availability, water, food), histone deacetylation plays an important role. In mammals, hibernation is a hypometabolic process in response to temperature, food and other environmental changes. During hibernation, it was shown that histone $\mathrm{H} 3$ acetylation and RNA polymerase II transcriptional activity decreases with an accompanying increase in HDAC I and IV 
protein levels ${ }^{6}$.

Although many of the environmental stresses listed above do not apply to humans, food is a common environmental stress in our society given the prevalence of eating disorders. Therefore, caloric restriction experiments have been conducted to investigate the epigenetic basis of metabolism. A specific class of HDACs (sirtuins) have been identified to play a significant role in regulating glucose and lipid metabolism during caloric restriction? ${ }^{7}$. The changes to histone deacetylation in response to environmental stress have formed the basis of our understanding of transcriptional silencing due to HDACs. This knowledge is paving the way for more clinically-relevant studies of histone deacetylation.

\section{HDACs and Disease}

Given the widespread and profound impact of epigenetic modifications to affect most, if not all physiological processes, HDACs have been studied in relation to a multitude of diseases ${ }^{3,8,9}$. For the onset and progression of cancer, research has shown that HDACs can inhibit the transcription of tumour-suppressor and apoptotic genes, thereby promoting tumour development ${ }^{3}$ (Figure 1). Similarly during cardiac hypertrophy and heart disease, the expression of a large number of genes is altered, and HDACs were shown to be involved regulating the expression of these genes ${ }^{8}$.

One disease that is being studied in relation to HDACs with increasing frequency is Spinal Muscular Atrophy (SMA). This life-threatening disease is a neuromuscular disorder characterized by motor neuron loss due to reduced survival of motor neuron (SMN) protein levels, and an increase in muscle atrophy. Thus children with the disease have significant impairments in mobility, leading to death in severe cases. HDACs have been shown to reduce SMN levels and promote muscle atrophy ${ }^{9}$. Therefore, the dual role that HDACs play in SMA pathology makes HDAC inhibitors a promising therapeutic.

\section{Clinical Implications of Histone Deacetylation - HDAC Inhibitors as a Therapeutic}

As mentioned previously, HDAC inhibitors are being studied extensively as a potential therapeutic for SMA, with Trichostatin A (an HDAC inhibitor) having been shown to ameliorate motor neuron and muscle growth ${ }^{9,10}$.

With respect to cancer, HDAC inhibitors are highly effective in selectively targeting tumour cells, and while these drugs have potent effects when administered alone, their combination with other anticancer agents (e.g. retinoic acid, UV irradiation) have produced even greater results ${ }^{3}$.

Presently, Vorinostat and romidepsin are two HDAC inhibitors that have already went through clinical trials and are Food and Drug Administration (FDA) approved for anticancer treatments with many others currently undergoing clinical trials for various diseases ${ }^{3,8}$. Therefore, the clinical applications of epigenetics-based therapeutics are very promising.

\section{Conclusion}

In contrast with changes in the genome, epigenetic changes such as histone deacetylation occur much more frequently in response to changes in the environment ${ }^{6,7}$, thus adding further complexity to the expression of genes. The clinical implications of epigenetic modifications have already been studied extensively in relation to HDAC, with promising results that have lead to FDA approval on two HDAC inhibitors ${ }^{8}$. Current HDAC inhibitors either inhibit specific classes of HDACs or are global inhibitors ${ }^{3}$. Therefore, understanding the differential modification of specific histone and non-histone residues in disease, and the development of therapeutics to target these specific modifications will bring us closer to providing personalized gene therapies.

\section{References}

1. Wolffe AP, Matzke MA. Epigenetics: regulation through repression. Science 1999 Oct 15;286(5439):481-486.

2. Macaluso M, Montanari M, Cinti C, Giordano A. Modulation of cell cycle components by epigenetic and genetic events. Semin Oncol 2005 Oct;32(5):452-457.

3. Bolden JE, Peart MJ, Johnstone RW. Anticancer activities of histone deacetylase inhibitors. Nat Rev Drug Discov 2006 Sep;5(9):769-784.

4. Spange $\mathrm{S}$, Wagner $\mathrm{T}$, Heinzel $\mathrm{T}$, Kramer $\mathrm{OH}$. Acetylation of non-histone proteins modulates cellular signalling at multiple levels. Int J Biochem Cell Biol 2009 Jan;41(1):185-198.

5. Ellis DJ, Lawman ZK, Bonham K. Histone acetylation is not an accurate predictor of gene expression following treatment with histone deacetylase inhibitors. Biochem Biophys Res Commun 2008 Mar 14;367(3):656-662.

6. Morin P,Jr, Storey KB. Evidence for a reduced transcriptional state during hibernation in ground squirrels. Cryobiology 2006 Dec;53(3):310-318.

7. Auwerx J, Houtkooper RH, Pirinen E. Sirtuins as regulators of metabolism and healthspan. Nature Reviews Molecular Cell Biology 2012 04; 2015/1;13:225+

8. Abend A, Kehat I. Histone deacetylases as therapeutic targets - From cancer to cardiac disease. Pharmacol Ther 2014 Nov 6.

9. Bricceno KV, Sampognaro PJ, Van Meerbeke JP, Sumner CJ, Fischbeck $\mathrm{KH}$, Burnett BG. Histone deacetylase inhibition suppresses myogenindependent atrogene activation in spinal muscular atrophy mice. Hum Mol Genet 2012 Oct 15;21(20):4448-4459. 
10. Liu H, Yazdani A, Murray LM, Beauvais A, Kothary R. The Smn-independent beneficial effects of trichostatin $A$ on an intermediate mouse model of spinal muscular atrophy. PLoS One 2014 Jul 1;9(7):e101225.

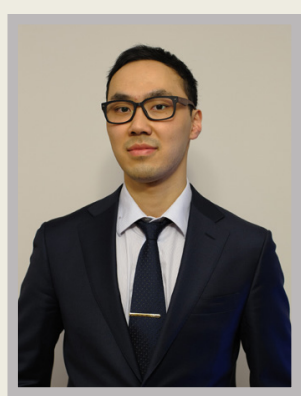

\section{Yichi (Tony) Zhang}

Tony Zhang completed his B.Sc. in Kinesiology at Queen's University and is currently entering the final year of his M.Sc. at Carleton University in the Department of Biology. His research focuses on a comparison between skeletal and cardiac muscle in thirteen-lined ground squirrels. These animals hibernate during winter months, where they demonstrate a remarkable capability to preserve their muscle mass despite prolonged periods of inactivity. They also undergo reversible cardiac hypertrophy during hibernation. Understanding these physiological adaptations to stress in ground squirrels has clinical implications in developing new treatments for muscle-wasting diseases as well as heart failure. Aside from research, Tony is involved in various aspects of athletics, from officiating basketball to rowing for the Ottawa Rowing Club and Carleton University. 